CIRJE-F-874

\title{
A New Improvement Scheme for Approximation Methods of Probability Density Functions
}

\author{
Akihiko Takahashi \\ University of Tokyo \\ Yukihiro Tsuzuki \\ Graduate School of Economics, University of Tokyo \\ January 2013
}

CIRJE Discussion Papers can be downloaded without charge from:

http://www.cirje.e.u-tokyo.ac.jp/research/03research02dp.html

Discussion Papers are a series of manuscripts in their draft form. They are not intended for circulation or distribution except as indicated by the author. For that reason Discussion Papers may not be reproduced or distributed without the written consent of the author. 


\title{
A New Improvement Scheme for Approximation Methods of Probability Density Functions*
}

\author{
Akihiko Takahashi† Yukihiro Tsuzuki ${ }^{\ddagger}$ \\ First Version: November 18,2012 , This Version: January 12, 2013
}

\begin{abstract}
This paper develops a new scheme for improving an approximation method of a probability density function, which is inspired by the idea in best approximation in an inner product space. Moreover, we applies "Dykstra's cyclic projections algorithm" for its implementation. Numerical examples for application to an asymptotic expansion method in option pricing demonstrate the effectiveness of our scheme under BlackScholes and SABR models.
\end{abstract}

Keywords: Density approximation, Probability density function, Asymptotic expansion, Best approximation in inner product spaces, Dykstra's algorithm, Option pricing, SABR model

${ }^{*}$ All the contents expressed in this research are solely those of the authors and do not represent the view of any institutions. The authors are not responsible or liable in any manner for any losses and/or damages caused by the use of any contents in this research.

${ }^{\dagger}$ Graduate School of Economics, University of Tokyo

${ }^{\ddagger}$ Graduate School of Economics, University of Tokyo, E-mail: yukihirotsuzuki@gmail.com 


\section{Introduction}

An approximation for a probability density function is a very interesting topic in various research fields. In fact, it seems so useful that a precise analytical approximation for a density would lead to substantial reduction of computational burden so that the subsequent analyses could be very easily implemented. Particularly, in finance the approximations for the densities of the asset prices have drawn much attention for at least more than two decades since fast and precise computation is so important in terms of competition and risk management: for instance, it is crucial in evaluation of derivatives.

An example among a large number of the related researches is an asymptotic expansion approach initiated by [19] Yoshida [63] and [41], which is mathematically justified by Watanabe theory (Watanabe [59]) in Malliavin calculus (e.g. Malliavin [28], Chapter V-8 in Ikeda and Watanabe [17], Nualart [32]). Actually, the asymptotic expansion have been applied to a broad class of problems in finance: See for instance, [42], [43], [20], [21], [22], [30], [35], [36], [37], [38], [39], [44], [45], [53], [54] [56], [57], [52], [46], [47], [48], Li [27], Osajima [33].

For other approximation methods in mathematical finance/financial engineering see for example, Bayer and Laurence [3], Ben Arous and Laurence [4], Gatheral, Hsu, Laurence, Ouyang, and Wang [14], Fouque, Papanicolaou and Sircar [13], Henry-Labordere [25], Kusuoka and Osajima [23], Osajima [34], Siopacha and Teichmann [40], and [10], [11], [12], [18], [55], [60], [61].

Although the asymptotic expansion up to the fifth order is known to be sufficiently accurate for option pricing (e.g. [37], [49], [50], [51]), one of the main criticisms against the method would be that the approximate density function admits negative values typically at its tails that is, some region of the deep Out-of-The-Money (OTM), which could create an arbitrage opportunity in option trading. Also, even if the domain of a true density is restricted to be positive, the domain of its approximation may include negative values unless an appropriate boundary condition is assigned. However, it seems that those and some other problems exist, at least implicitly in other approximation methods.

This paper develops a new scheme for improving density approximation methods, which also contributes to precise approximation of option values efficiently. Specifically, our scheme is inspired by the idea in $a$ best approximation method in an inner product space, and so called "Dykstra's cyclic projections algorithm" is applied for its implementation.

Firstly, we introduce an inner product with some appropriate weight function: the function is in fact a density of a certain probability measure. Then, we represent a closed convex space satisfying the required conditions in terms of the inner product. Thus, we are able to obtain the unique best approximation from a given approximate density to the space in terms of the norm induced by the inner product, which is also shown to be a better approximation for the true density than the original one measured by the norm.

More concretely, we put two basic conditions for a density function such that its total mass is one and its range is nonnegative. It should be noted that if we consider the density of the underlying asset price under a risk-neutral or a forward probability measure, the nonnegative density is necessary for arbitrage-free option prices.

Also, we put calibration conditions for the underlying asset prices and option values. That is, the average value under the approximate density of the underlying asset price at maturity should be equal to the current underlying forward price, which is generally known in advance. Moreover, as it is known that the asymptotic expansion method gives precise approximate values of the options close to ATM, a new approximate density may be matched to the density obtained by the asymptotic expansion method for a certain range close to ATM. In addition, option prices around ATM based on the asymptotic expansion method may be used for calibration by a new approximate density.

Further, if we have some information on the tail behavior of the target density such as those in Lee [26] and Benaim et al. [5], we may set tail conditions which will be given concretely in the next section.

We reflect those conditions in terms of an appropriate inner product space. Then, by using the corresponding norm we implement the best approximation from the density obtained by the asymptotic expansion to the closed convex space created by the required conditions. Consequently, we get a better estimate (in 
terms of the norm) for the true density of the underlying asset price than the one based on the original asymptotic expansion. In computation, we utilize so called Dykstra's cyclic projections algorithm. (e.g. see pp.207-214 of Deutsch [8] for the detail.)

Furthermore, numerical experiments for pricing plain-vanilla options under Black-Scholes([6]) and SABR ([15], [16]) models demonstrate the validity of our scheme. In fact, our scheme improves the third order asymptotic expansion preserving the required conditions such as nonnegative densities under an appropriate forward measure.

We finally remark that our scheme is general and flexible enough to be applied to approximation methods other than the asymptotic expansion approach. For example, it is well known that the density of the approximation formula for SABR model (Hagan et al. [15], [16]) has the negative values for the left tail. Hence, a number of researches have been going on in order to extend SABR model with fixing the problem of the negative densities. (For instance, see [1], [2], [9].) we note that our scheme is a candidate for handling this issue, too.

The organization of the paper is as follows: After the next section describes the setup of the problem, Section 3 provides a concrete formulation of our method as well as the algorithm for the implementation. Section 4 shows numerical examples under Black-Scholes and SABR models. Section 5 concludes.

\section{Setup}

Let $S_{t}$ be the spot price of the underlying asset at time $t \in[0, T]$ and consider a density $f$ of $S_{T}$, where $S_{T}$ takes a value in $I \subseteq \mathbb{R}$, such as $I=\mathbb{R},[0,+\infty)$ or $(0,+\infty)$. Clearly, the density function $f$ of the price $S_{T}$ must satisfy the following property. Hereafter, $\eta$ stands for a density function under a risk-neutral or an appropriate forward probability measure.

Property 1. (Density Condition) : for a function $\eta$ on $I \subseteq \mathbb{R}$,

(1) $\int_{I} \eta(x) d x=1$

(2) $\eta \geq 0$.

Suppose that we have an approximation $\tilde{f}$ of the density function $f$ by some method such as asymptotic expansion scheme. Note that the approximation $\tilde{f}$ does not necessarily have the properties 1 . Actually, it has been found through our numerical experience under Black-Scholes model that $\tilde{f}$ possibly takes a negative value at its tail, namely "deep out of the money."

Also, generally the forward price is given independently of models, and hence the average value of the underlying asset price at $T$ should be equal to the given forward price with maturity $T$. Moreover, it is known that the asymptotic expansion method provides rather precise approximations for the values close to At-The-Money(ATM) options. Thus, it is reasonable that the option prices around ATM under a new approximate density function are calibrated to those computed based on the asymptotic expansion, and that a new density is equal to the one obtained by the asymptotic expansion for a certain range around ATM of the underlying asset price. We call those properties by Calibration Condition:

Property 2. (Calibration Condition)

(3) $\int_{I} x \eta(x) d x=S_{0}$

(4) $\int_{I}\left(x-K_{n}\right)_{+} \eta(x) d x=C_{K_{n}}$ for some given strikes $\left\{K_{n}\right\}_{n=1}^{N}$

(5) $\eta=\tilde{f}$ on some subset $I_{0}$ of $I$

Here, the risk-free interest rate as well as the dividend rate of the underlying asset are assumed to be zero for simplicity. $C_{K_{n}}$ denotes the option price with strike $K_{n}$ and maturity $T$ computed by the asymptotic expansion method. 
In contrast to the accuracy around ATM, the values of the approximated density $\tilde{f}$ may not be reliable around deep out of the money. However, how fast a density decreases to zero is known under some models or through a moment formula for the implied volatility. Namely, the following quantities are known:

$$
\begin{aligned}
& \tilde{p}:=\sup \left\{p>0: \mathbb{E} S_{T}^{p}<+\infty\right\} \\
& \tilde{q}:=\sup \left\{q>0: \mathbb{E} S_{T}^{-q}<+\infty\right\}
\end{aligned}
$$

under some models or through the moment formula derived by Lee [26]:

$$
\tilde{p}=\frac{1}{2 \beta_{R}}+\frac{\beta_{R}}{8}+\frac{1}{2}, \quad \tilde{q}=\frac{1}{2 \beta_{L}}+\frac{\beta_{L}}{8}-\frac{1}{2},
$$

where

$$
\begin{aligned}
& \beta_{R}:=\lim \sup _{x \rightarrow+\infty} \frac{I V^{2}(x)}{|x| / T}, \\
& \beta_{L}:=\lim \sup _{x \rightarrow-\infty} \frac{I V^{2}(x)}{|x| / T} .
\end{aligned}
$$

Here, $I V(x)$ is an implied volatility function in terms of the log-moneyness that is, $x=\log \left(S_{0} / K\right)$.

Now, let us assume that $\tilde{p}$ and $\tilde{q}$ are known, and suppose $\chi: I \longrightarrow(0,+\infty)$ be a density function which has the same order of the tail condition as $f$ :

$$
\int_{S_{0}}^{+\infty} x^{p} \chi(x) d x<+\infty \quad(p<\tilde{p}), \quad \int_{0}^{S_{0}} x^{-q} \chi(x) d x<+\infty \quad(q<\tilde{q}) .
$$

Then, it seems natural to impose the following condition:

Property 3. (Tail Condition)

(6) $\eta$ has the same tail slopes as $\chi$.

However, for ease of computation, the condition may be replaced with the following:

Property 4. (Weak Tail Condition)

(6-1) $\eta \leq \chi$ on $\left(0, K_{L}\right]$ for some positive number $K_{L}$

(6-2) $\eta \leq \chi$ on $\left[K_{R},+\infty\right)$ for some positive number $K_{R}$

Thus, we state our problem formally as follows:

Definition 1 (Problem). Find a new approximate density function $f^{*}$ for the target density $f$ such that it satisfies the properties 1, 2 and 4, and

$$
\left\|f-f^{*}\right\| \leq\|f-\tilde{f}\|,
$$

where the norm $\|\cdot\|$ will be defined in the next section.

\section{Formulation and Algorithm}

This section concretely formulates the previous discussion and provides an algorithm for the implementation. 


\subsection{Formulation}

Firstly, suppose a probability space $(\mathbb{R}, \mathcal{M}, \mu)$, where the measure $\mu$ is assumed to have a density which is equal to $\chi$ given in the previous section on $I$ and to 0 on $I^{c}$. Next, we define the set of square integrable functions on $(\mathbb{R}, \mathcal{M}, \mu)$ denoted by $\mathcal{H}:=L^{2}(\mathbb{R}, \mathcal{M}, \mu)$, and introduce an inner product for $f, g \in \mathcal{H}$ by

$$
\langle f, g\rangle=\int_{I} f(x) g(x) \chi(x) d x .
$$

With this preparation, the properties (1) to (5), (6-1) and (6-2) in Section 2 are restated as follows:

(1) $\left\langle\eta / \chi, 1_{I}\right\rangle=1$

(2) $\left\langle\eta / \chi, \delta_{x}\right\rangle \geq 0$ for each $x \in I$

(3) $\left\langle\eta / \chi, i d_{I}\right\rangle=S_{0}$

(4) $\left\langle\eta / \chi, g_{K_{n}}\right\rangle=C_{K_{n}}$

(5) $\left\langle\eta / \chi, \delta_{x}\right\rangle=\tilde{f}(x)$ for each $x \in I_{0}$

(6-1) $\left\langle\eta / \chi, \delta_{x}\right\rangle \leq \chi(x)$ for each $x \in\left(0, K_{L}\right]$

(6-2) $\left\langle\eta / \chi, \delta_{x}\right\rangle \leq \chi(x)$ for each $x \in\left[K_{R},+\infty\right)$,

where $1_{I}, i d_{I}$ and $g_{K_{n}}$ are elements of $\mathcal{H}$ such that $1_{I}(x)=1, i d_{I}(x)=x$ and $g_{K_{n}}(x)=\left(x-K_{n}\right)_{+}$.

Next, let us define some subsets of $\mathcal{H}$ as $\mathcal{K}_{D}, \mathcal{K}_{C}$ and $\mathcal{K}_{T}$ which stand for the properties (1)-(2), (3)-(5) and (6-1)-(6-2) above, respectively:

$$
\mathcal{K}_{D}:=\left\{\varphi \in \mathcal{H} \mid\left\langle\varphi, 1_{I}\right\rangle=1\right\} \cap \bigcap_{x \in I}\left\{\varphi \in \mathcal{H} \mid\left\langle\varphi, \delta_{x}\right\rangle \geq 0\right\},
$$

$$
\mathcal{K}_{C}:=\left\{\varphi \in \mathcal{H} \mid\left\langle\varphi, i d_{I}\right\rangle=S_{0}\right\} \cap \bigcap_{n \leq N}\left\{\varphi \in \mathcal{H} \mid\left\langle\varphi, g_{K_{n}}\right\rangle=C_{K_{n}}\right\} \cap \bigcap_{x \in I_{0}}\left\{\varphi \in \mathcal{H} \mid\left\langle\varphi, \delta_{x}\right\rangle=\tilde{f}(x)\right\}
$$

and

$$
\mathcal{K}_{T}:=\bigcap_{x \in\left(0, K_{L}\right]}\left\{\varphi \in \mathcal{H} \mid\left\langle\eta / \chi, \delta_{x}\right\rangle \leq \chi(x)\right\} \cap \bigcap_{x \in\left[K_{R},+\infty\right)}\left\{\varphi \in \mathcal{H} \mid\left\langle\eta / \chi, \delta_{x}\right\rangle \leq \chi(x)\right\}
$$

Moreover, define $\mathcal{K}$ as the intersection of $\mathcal{K}_{D}, \mathcal{K}_{C}$ and $\mathcal{K}_{T}$ :

$$
\mathcal{K}:=\mathcal{K}_{D} \cap \mathcal{K}_{C} \cap \mathcal{K}_{T},
$$

which is assumed to be nonempty.

Then, let $\tilde{\varphi}:=\tilde{f} / \chi \notin \mathcal{K}$, and the best approximation set from $\tilde{\varphi}$ to $\mathcal{K}$ is defined as

$$
P_{\mathcal{K}}(\tilde{\varphi}):=\left\{\varphi^{*} \in \mathcal{K} \mid\left\|\tilde{\varphi}-\varphi^{*}\right\|=\inf _{\eta \in \mathcal{K}}\|\tilde{\varphi}-\eta\|\right\} .
$$

Note that the set $P_{\mathcal{K}}(\tilde{\varphi})$ has the only one element since $\mathcal{K}$ is a closed convex set in a Hilbert space. Hereafter, we may use the notation $P_{\mathcal{K}}(\tilde{\varphi})$ for the unique element of $P_{\mathcal{K}}(\tilde{\varphi})$ without any confusion.

Thus, it is easily shown that $P_{\mathcal{K}}(\tilde{\varphi})$ is a better approximation for $f / \chi \in \mathcal{K}$ than $\tilde{\varphi}:=\tilde{f} / \chi \notin \mathcal{K}$, because we have

$$
\begin{aligned}
\left\|f / \chi-P_{\mathcal{K}}(\tilde{\varphi})\right\|^{2} & =\left\|f / \chi-\varphi^{\perp}\right\|^{2}+\left\|\varphi^{\perp}-P_{\mathcal{K}}(\tilde{\varphi})\right\|^{2} \\
& \leq\left\|f / \chi-\varphi^{\perp}\right\|^{2}+\left\|\varphi^{\perp}-\tilde{\varphi}\right\|^{2} \\
& =\|f / \chi-\tilde{\varphi}\|^{2}
\end{aligned}
$$


where $\varphi^{\perp}$ is the foot of a perpendicular line through $\tilde{\varphi}$ and $P_{\mathcal{K}}(\tilde{\varphi})$ from $f / \chi$.

Finally, we are able to obtain a better approximated density function as

$$
f^{*}:=P_{\mathcal{K}}(\tilde{\varphi}) \chi .
$$

This is actually better than the original one $\tilde{f}$ in the following sense:

$$
\int_{I}\left|f(x)-f^{*}(x)\right|^{2} \frac{1}{\chi(x)} d x \leq \int_{I}|f(x)-\tilde{f}(x)|^{2} \frac{1}{\chi(x)} d x .
$$

\subsection{Algorithm}

In order to compute (3.5), we applies an iterative algorithm called Dykstra's algorithm. (See pp. 207-214 of Deutsch [8] for the detail of the algorithm and its convergence discussion.)

In particular, let $\tilde{\mathcal{K}}$ be a convex set obtained by discretization of $\mathcal{K}$, which is an intersection of finite many closed convex sets $\mathcal{K}_{i}(i=1,2, \cdots, r)$ in the Hilbert space $\mathcal{H}$ :

$$
\tilde{\mathcal{K}}=\cap_{i=1}^{r} \mathcal{K}_{i} .
$$

Here, we assume $\tilde{\mathcal{K}}$ to be nonempty.

First, for each $n \in \mathbb{N}$, let $[n]$ denote $n \bmod r$; that is,

$$
[n]:=\{1,2, \cdots, r\} \cap\{n-k r: k=0,1,2, \cdots\} .
$$

For instance, $[1]=1,[2]=2, \cdots,[r]=r,[r+1]=1, \cdots,[2 r]=r, \cdots$.

Next, for $\tilde{\varphi} \in \mathcal{H}$, set

$$
\begin{aligned}
\varphi_{0} & :=\tilde{\varphi}, e_{-(r-1)}=\cdots=e_{-1}=e_{0}=0, \\
\varphi_{n} & :=P_{\mathcal{K}_{[n]}}\left(\varphi_{n-1}+e_{n-r}\right), \\
e_{n} & :=\varphi_{n-1}+e_{n-r}-\varphi_{n} \\
& =\varphi_{n-1}+e_{n-r}-P_{\mathcal{K}_{[n]}}\left(\varphi_{n-1}+e_{n-r}\right) .
\end{aligned}
$$

Then, we have

$$
\lim _{n \rightarrow+\infty}\left\|\varphi_{n}-P_{\mathcal{K}}(\tilde{\varphi})\right\|=0
$$

See Theorem 9.24 (Boyle-Dykstra Theorem) in p. 213 of Deutsch [8] for the proof.

\section{Numerical Example}

This section examines the validity of our method through numerical experiments under Black-Scholes and SABR models.

\subsection{Black-Scholes Model}

Firstly, let us consider Black-Scholes model, where an analytical formulas for the density of the underlying asset price and option values are known. Under Black-Scholes model, the dynamics of the underlying price process under an appropriate forward measure is given as follows:

$$
d S_{t}=\sigma S_{t} d W_{t}
$$


where $\sigma$ is a constant. Clearly, the density function of $S_{T}, f(x)$ is given by a log-normal:

$$
f(x)=\frac{1}{\sqrt{2 \pi}} \frac{1}{x \sigma \sqrt{T}} e^{-\frac{(\log x-m)^{2}}{2 \sigma^{2} T}},
$$

where $m:=\log S_{0}-\frac{1}{2} \sigma^{2} T$. For this case, we take $\chi(x)$ for $I:=(0,+\infty)$ as follows:

$$
\chi(x)= \begin{cases}A_{L} \frac{1}{\sqrt{2 \pi}} \frac{1}{x \sigma \sqrt{T}} e^{-\frac{(\log x-m)^{2}}{2 \sigma^{2} T}} & x \in\left(0, K_{L}\right) \\ f_{3}(x) & x \in\left[K_{L}, K_{R}\right) \\ A_{R} \frac{1}{\sqrt{2 \pi}} \frac{1}{x \sigma \sqrt{T}} e^{-\frac{(\log x-m)^{2}}{2 \sigma^{2} T}} & x \in\left[K_{R},+\infty\right),\end{cases}
$$

and $K_{L}, K_{R}, A_{L}$ and $A_{R}$ positive constants and $f_{3}$ stands for the density function obtained by the third order asymptotic expansion.

With this setup, we calculate option values in the three different ways below:

(a) exact Black-Scholes formula (Benchmark)

(b) asymptotic expansion up to the third order

(c) our algorithm with $K_{i}=80,100,120\left(N=3\right.$ in $\mathcal{K}_{C}$ of $\left.(3.2)\right)$ and $f^{*}=\tilde{f}$ on $[70,150)$,

where we set $\tilde{f}=f_{3}$ and specify Black-Scholes model parameters as $S_{0}=100, \sigma=0.15, T=2, K_{L}=70$ and $K_{R}=150 ; A_{L}$ and $A_{R}$ are determined so that $\chi$ is continuous at $K_{L}$ and $K_{R}$, respectively.

The results for the option prices and the density functions obtained by three different methods above are shown in Table 2 and Figure 1-3, respectively.

In the table, the comparison between the third order expansion (b) and our method (c) shows that our method improves accuracies for the deep OTM prices, where the strike prices are in the range of 10-50 and 160-200. On the other hand, for the strikes in the range of $60-150$, both methods provide the same order of good accuracies: it is plausible because in our method (c) the option values around ATM and the density between 70 and 150 of the underlying asset price are calibrated to those based on the third order expansion.

Although Figure 1 shows that both the third order expansion and our method produce good approximations in total, the close look at the left tail in Figure 2 reveals that the third order expansion suffers the negative densities in the range of 31-48 of the underlying asset price. It also puts very small but nonzero densities on the negative underlying asset values, which is not the case for the log-normal model with a positive initial value.

On the other hand, our method keeps the density nonnegative due to the property (2) in Section 3, reflected in $\mathcal{K}_{D}$ of (3.1). Moreover, by construction it does not put any densities on the negative underlying asset prices.

Furthermore, Figure 3 for the right tail of the density clarifies that our method improves the approximation, especially around 160 of the underlying price.

Consequently, we can conclude that our scheme works very well for this case.

\subsection{SABR Model}

The second example takes SABR model as a stochastic volatility model, where the underlying price process under a forward measure follows:

$$
\begin{aligned}
d S_{t} & =\sigma_{t} S_{t}^{c} d W_{t}^{1} \\
d \sigma_{t} & =\varepsilon \sigma_{t} d W_{t}^{2},
\end{aligned}
$$

where $\varepsilon>0, c \in(0,1]$ and $W^{1}$ and $W^{2}$ are Brownian motions with a constant correlation $\rho$. Firstly, let us consider the case for $c=1$ and $\rho<0$. According to the result by Benaim et al. [5], it holds that in

$$
\tilde{p}=\frac{1}{1-\rho^{2}}, \quad \tilde{q}=0 .
$$


In this case, the left tail is so fat that any moment is infinite, that is $\mathbb{E}\left(S_{T}^{-q}\right)=+\infty$ for $q>0$. Hence, taking the observation into consideration, we may set $\chi$ for $I:=(0,+\infty)$ as follows:

$$
\chi(x)= \begin{cases}\gamma x+A_{L} & x \in\left(0, K_{L}\right) \\ f_{3}(x) & x \in\left[K_{L}, K_{R}\right) \\ \beta(x-\alpha)^{-\tilde{p}} & x \in\left[K_{R},+\infty\right)\end{cases}
$$

where $K_{L}, K_{R}, A_{L}, \alpha, \beta$ and $\gamma$ are positive constants and $f_{3}$ stands for the density function obtained by the third order asymptotic expansion.

Next, let us consider the case for $c<1$. For this case, it is known that $S_{T}$ can reach 0 with positive probability. According to the result by Benaim et al. [5], it holds that

$$
\tilde{p}=+\infty, \quad \tilde{q}=0 .
$$

Then, while the density decreases so fast to zero on the right tail, the left tail is so fat that any moment is infinite: $\mathbb{E}\left(S_{T}^{p}\right)<+\infty$ for $p>0$ and $\mathbb{E}\left(S_{T}^{-q}\right)=+\infty$ for $q>0$. Thus, taking this observation into account, we may specify $\chi$ for $I:=[0,+\infty)$ in the following:

$$
\chi(x)= \begin{cases}A_{L} & x=0, \\ f_{3}(x) & x \in\left(0, K_{R}\right) \\ A_{R} \frac{1}{\sqrt{2 \pi}} \frac{1}{x \tilde{\sigma} \sqrt{T}} e^{-\frac{(\log x-m)^{2}}{2 \tilde{\sigma}^{2} T}} & x \in\left[K_{R},+\infty\right),\end{cases}
$$

where $A_{L}$ is a positive constant and $m=\log S_{0}-\frac{1}{2} \tilde{\sigma}^{2} T$. Note that we do not impose the weak tail condition (6-1) for this case.

With this setup, we calculate OTM option prices by the following methods:

(a) Monte Carlo simulation (Benchmark)

(b) asymptotic expansion up to the fifth order

(c) asymptotic expansion up to the third order

(d) our algorithm with $K_{i}=80,100,120\left(N=3\right.$ in $\mathcal{K}_{C}$ of $\left.(3.2)\right)$, and $f^{*}=\tilde{f}$ on $\left[K_{L}, K_{R}\right)$ for $c=1$ and on $\left(0, K_{R}\right)$ for $c=\frac{1}{2}$, where $\tilde{f}$ is equivalent to (c) the third order expansion.

For both cases of $c=1$ and $c=\frac{1}{2}$, we set $\tilde{f}=f_{3}, K_{R}=150$ and $A_{L}=\tilde{f}(0)$. Moreover, for the case of $c=1$, we set $K_{L}=50 ; \gamma$ is determined so that $\chi$ is continuous; $\alpha$ and $\beta$ are determined by $\chi(x)=\tilde{f}(x)$ for some two points around $x=K_{R}$ so that the function $\chi$ decreases so fast to zero as the function $\tilde{f}$ does around $x=K_{R}$. For the case of $c=\frac{1}{2}$, we set $\tilde{\sigma}=0.45$, which is the same level as the implied volatility around $K_{R} ; A_{R}$ is determined so that $\chi$ is continuous. We set the other parameters as in Table 1 below.

The results for the option values by four methods (a)-(d) above are given in Table 3 for $c=1$ and Table 4 for $c=\frac{1}{2}$. Also, the density functions obtained by four methods (a)-(d) are shown in Figure $4-6$ for $c=1$ and Figure 7 - 9 for $c=\frac{1}{2}$.

Table 3 for $c=1$ shows that comparing to the third order expansion (c), our method (d) provides the better approximations for 12 out of 20 cases: it substantially improves the accuracies for the deep OTM prices for the strikes in the range of 10-50 and 160-200, while the accuracies of (c) and (d) are comparable in the range of 60-150, which is expected because in our method (d) the option values around ATM and the density between 50 and 150 of the underlying asset price are calibrated to those based on the third order expansion.

On the other hand, the fifth order expansion (b) still produces the best approximations, which is reasonable since our new method is designed based on the third order expansion. In terms of the density approximations, although Figure 5 shows that our method and the fifth order expansion look better than the third order expansion in total, the examination of the left tail in Figure 5 reveals that the fifth order expansion suffers the negative densities in some range below 5 of the underlying asset price, while our method 
does not suffer those for all the range by construction. Also, as in Black-Scholes model, the third and fifth order expansions put some nonzero (negative) densities on the negative values of the underlying asset price, which is not the case for the benchmark Monte Carlo and our method.

On the other hand, Figure 6 shows that the fifth order expansion has no problem for the right tail, and our method improves the third order expansion around between 160 and 220 of the underlying asset price.

Moreover, we expect that applying our method to the fifth order expansion will improve the approximation accuracies of option prices with the conditions such as the nonnegative densities and no densities on the negative underlying asset values.

In the approximation of the option values for $c=\frac{1}{2}$, Table 4 confirms that the similar observation holds as for $c=1$, except that our method improves the third order expansion more substantially than for $c=1$, and produces sufficient accuracies comparable to the fifth order expansion.

Further, thanks to the flexibility of our choice of $\chi(x)$ in (4.9), our density approximation is able to take the absorption barrier at $x=0$ into consideration, at least to a certain extent, which cannot be achieved by the original asymptotic expansion method only: the asymptotic expansion itself puts nonzero densities on some negative values of the underlying asset prices as in the cases of Black-Scholes and SABR with $c=1$. We observe these points in Figure 7 - 9. Note finally that the approximation is expected to become more precise if we can add other accurate information such as for the probability of $S_{T}=0$ (e.g. Doust [9]) to $\mathcal{K}$ in (3.4) through its inner product expression.

Table 1: Parameter

\begin{tabular}{cccccc}
\hline$S_{0}$ & $c$ & $\sigma_{0}$ & $\varepsilon$ & $\rho$ & $T$ \\
\hline 100 & 0.5 or 1.0 & 0.5 & 0.3 & -0.5 & 1 \\
\hline
\end{tabular}

\section{Conclusion}

We have proposed a new improvement scheme of approximation methods for probability density functions, of which applications contain option pricing and computation of Greeks in finance.

Particularly, we have made use of an idea of "best approximation in an inner product space from a convex set," and applied an iterative algorithm called Dykstra's algorithm for its implementation. Consequently, we have obtained a better approximate density that satisfies a set of appropriate conditions including the nonnegative density under a risk-neutral or a forward measure necessary for no arbitrage option prices.

Moreover, an application to an asymptotic method has confirmed the validity of our scheme through numerical experiments under Black-Scholes and SABR models.

We finally remark that our scheme is general and flexible enough to be applied to other density approximation methods such as Hagan et al. [16], as well as to include some other accurate information for the true density such as for the probability of the absorption at origin. Further applications and extensions along the line are among our next research topics.

\section{References}

[1] Andreasen, J. and Huge, B., [2011a], Volatility Interpolation, Risk Magazine, March 2011, 76-79.

[2] Andreasen, J. and Huge, B., [2011b], ZABR - Expansions for the Mass -, Preprint.

[3] Bayer, C., and Laurence, P., [2012], Asymptotics beats Mote Carlo: The case of correlated local vol baskets, preprint, forthcoming in Communications on Pure and Applied Mathematics. . 
[4] Ben Arous, G., Laurence, P. [2009], “ Second order expansion for implied volatility in two factor local stochastic volatility models and applications to the dynamic $\lambda$-SABR model," preprint.

[5] Benaim, S., Friz, P. and Lee, R. [2008], "On Black-Scholes Implied Volatility at Extreme Strikes," In "Frontiers in Quantitative Finance: Volatility and Credit Risk Modeling," (Cont, R. ed.), Wiley, 2008.

[6] Black, F. and Scholes, M. [1973], "The Pricing of Options and Corporate Liabilities," Journal of Political Economy 81 (3): 637?654.

[7] Cox, J. [1975], "Notes on Option Pricing I: Constant Elasticity of Diffusions." Unpublished draft, Stanford University, 1975.

[8] Deutsch, F. [2001], "Best Approximation in Inner Product spaces," Springer, New York, 2001.

[9] Doust, P. [2012], No-arbitrage SABR, The Journal of Computational Finance, Vol. 15, No. 3, Spring 2012.

[10] Fujii, M. and Takahashi, A. [2012a], Analytical Approximation for Non-linear FBSDEs with Perturbation Scheme, International Journal of Theoretical and Applied Finance, Vol. 15, No. 5, (2012a).

[11] Fujii, M. and Takahashi, A. [2012b], Perturbative Expansion Technique for Non-linear FBSDEs with Interacting Particle Method, Working paper, CARF-F-278, the University of Tokyo.

[12] Fujii, M. and Takahashi, A. [2012c], "Perturbative Expansion of FBSDE in an Incomplete Market with Stochastic Volatility," Quarterly Jornal of Finance Vol.2, No.3 (2012) 1250015, DOI:10.1142/S2010139212500152.

[13] Fouque, J. P., G. Papanicolaou and K. R. Sircar [2000], Derivatives in Financial Markets with Stochastic Volatility, Cambridge University Press.

[14] [2009], Gatheral, J., Hsu, E.P., Laurence, P., Ouyang, C. and Wang, T-H., "Asymptotics of implied volatility in local volatility models," Mathematical Finance, 22(4), 2012, 591-620.

[15] Hagan, P.S., Kumar, D., Lesniewskie, A.S., and Woodward, D.E. [2001], Probability Distribution in the SABR Model of Stochastic Volatility, Preprint.

[16] Hagan, P.S., Kumar, D., Lesniewskie, A.S., and Woodward, D.E. [2002], Managing Smile Risk, Willmott Magazine, 2002, 84-108.

[17] Ikeda, N. and Watanabe, S. [1989], Stochastic Differential Equations and Diffusion Processes, Second Edition, North-Holland/Kodansha, Tokyo.

[18] Kato, T., Takahashi, A., Yamada. T., An Asymptotic Expansion for Solutions of Cauchy-Dirichlet Problem for Second Order ParabolicPDEs and its Application to Pricing Barrier Options, Working paper, CARF-F-271, the University of Tokyo, 2012.

[19] Kunitomo, N. and Takahashi, A., Pricing Average Options, Japan Financial Review, Vol.14, 1992, 1-20(in Japanese).

[20] Kunitomo, N. and Takahashi, A. [2001], "The Asymptotic Expansion Approach to the Valuation of Interest Rate Contingent Claims," Mathematical Finance, Vol. 11, 117-151.

[21] Kunitomo, N. and Takahashi, A., [2003], On Validity of the Asymptotic Expansion Approach in Contingent Claim Analysis, Annals of Applied Probability, Vol.13(3), 914-95, .2003. 
[22] Kunitomo, N. and Takahashi, A., [2004], "Applications of the Asymptotic Expansion Approach based on Malliavin-Watanabe Calculus in Financial Problems," Stochastic Processes and Applications to Mathematical Finance, 195-232.

[23] Kusuoka S. and Osajima, Y. [2007], "A Remark on the Asymptotic Expansion of Density Function of Wiener Functionals," Preprint, Graduate School of Mathematical Sciences, the University of Tokyo.

[24] Kusuoka S. and Strook, D. [1991], "Precise Asymptotics of Certain Wiener Functionals," Journal of Functional Analysis, Vol.99, 1-74.

[25] Henry-Labordère, P., Analysis, Geometry and Modeling in Finance : Advanced Methods in Options Pricing, Chapman and Hall, 2008.

[26] Lee, R. [2004], "The Moment Formula for Implied Volatility at Extreme," Mathematical Finance, 14(3): 469-480.

[27] Li, C. [2010], "Closed-form Expansion, Conditional Expectation, and Option Valuation," Guanghua School of Management, Peking University, Beijing, China.

[28] Malliavin, P. [1997], "Stochastic Analysis," Springer.

[29] Malliavin, P. and Thalmaier, A. [2006], Stochastic Calculus of Variations in Mathematical Finance, Springer.

[30] Matsuoka, R. Takahashi, A. and Uchida, Y. [2004], "A New Computational Scheme for Computing Greeks by the Asymptotic Expansion Approach," Asia-Pacific Financial Markets, Vol.11, 393-430.

[31] Nualart, D. [1995], "The Malliavin Calculus and Related Topics,"Springer.

[32] Nualart, D., Üstünel A. S. and Zalai M. [1988], "On the moments of a multiple Wiener-Itô integral and the space induced by the polynomials of the integral," Stochastics, Vol 25, 233-340.

[33] Osajima, Y. [2006], "The Asymptotic Expansion Formula of Implied Volatility for Dynamic SABR Model and FX Hybrid Model," Preprint, Graduate School of Mathematical Sciences, the University of Tokyo.

[34] Osajima, Y. [2007], "General Asymptotics of Wiener Functionals and Application to Mathematical Finance," Preprint, Graduate School of Mathematical Sciences, the University of Tokyo.

[35] Shiraya, K,, Takahashi, A., Pricing Average Options on Commodities, Journal of Futures Markets. Vol.31-5, 407-439, 2011.

[36] Shiraya, K, Takahashi, A. [2012], Pricing Multi-Asset Cross Currency Options, Working paper, CARFF-276, the University of Tokyo, 2012, forthcoming in Journal of Futures Markets.

[37] Shiraya, K., Takahashi, A., Toda, M., Pricing Barrier and Average Options under Stochastic Volatility Environment, Journal of Computational Finance vol.15-2, winter 2011/12, 111-148.

[38] Shiraya, K,, Takahashi, A., Yamazaki, A., Pricing Swaptions under the LIBOR Market Model of Interest Rates with Local-Stochastic Volatility Models, Forthcoming in "Wilmott Journal". 2010

[39] Shiraya, K,, Takahashi, A., Yamada, T. Pricing Discrete Barrier Options under Stochastic Volatility, Asia-Pacific Financial Markets Volume 19-3 , 205-232, 2012. 
[40] Siopacha, M. and Teichmann, J., "Weak and Strong Taylor Methods for Numerical Solutions of Stochastic Differential Equations," Quantitative Finance, Volume 11-4, 517-528, 2011.

[41] Takahashi, A., Essays on the Valuation Problems of Contingent Claims, Unpublished Ph.D. Dissertation, Haas School of Business, University of California, Berkeley, 1995.

[42] Takahashi, A., An Asymptotic Expansion Approach to Pricing Contingent Claims, Asia-Pacific Financial Markets, Vol. 6, 1999, 115-151.

[43] Takahashi, A. [2009], "On an Asymptotic Expansion Approach to Numerical Problems in Finance," Selected Papers on Probability and Statistics, pp. 199-217, 2009, American Mathematical Society.

[44] Takahashi, A. and Matsushima, S. [2004], "Monte Carlo Simulation with an Asymptotic Expansion in HJM Framework," FSA Research Review 2004, 82-103, Financial Services Agency (in Japanese).

[45] Takahashi, A. and Saito, T. [2003], "An Asymptotic Expansion Approach to Pricing American Options," Monetary and Economic Studies, Vol. 22, 35-87. (in Japanese).

[46] Takahashi, A. and Takehara, K.[2007], "Pricing Currency Options with a Market Model of Interest Rates under Jump-Diffusion Stochastic Volatility Processes of Spot Exchange Rates," Asia-Pacific Financial Markets, Vol.14, pp. 69-121.

[47] Takahashi, A. and Takehara, K.[2008], "Fourier Transform Method with an Asymptotic Expansion Approach: an Application to Currency Options," International Journal of Theoretical and Applied Finance, Vol.11-4, 381-401.

[48] Takahashi, A., Takehara, K. [2010], A Hybrid Asymptotic Expansion Scheme: an Application to Longterm Currency Options,', International Journal of Theoretical and Applied Finance, Vol.13-8, 11791221.

[49] Takahashi, A., Takehara, K. and Toda. M., Computation in an Asymptotic Expansion Method, Working paper, CARF-F-149, the University of Tokyo, 2009.

[50] Takahashi, A., Takehara, K. and Toda. M., A General Computation Scheme for a High-Order Asymptotic Expansion Method, International Journal of Theoretical and Applied Finance, 15(6), 2012.

[51] Takehara, K., Takahashi, A., and Toda. M., Application of a High-Order Aymptotic Expantion Scheme to Long-Term Currency Options, The International Journal of Business and Finance Research vol. 5-3, pp 87-100, 2011.

[52] Takahashi, A. and Uchida, Y. [2006], "New Acceleration Schemes with the Asymptotic Expansion in Monte Carlo Simulation," Advances in Mathematical Economics, Vol. 8, 411-431.

[53] Takahashi, A., Yamada, T., "An Asymptotic Expansion with Push-Down of Malliavin Weights," SIAM Journal on Financial Mathematics Volume 3, pp 95-136, 2012.

[54] Takahashi, A., Yamada, T., "A Remark on Approximation of the Solutions to Partial Differential Equations in Finance," Recent Advances in Financial Engineering 2011, pp.133-181, 2012.

[55] Takahashi, A., Yamada, T., "An Asymptotic Expansion for Forward-Backward SDEs: A Malliavin Calculus Approach," Preprint, CARF-F-296.

[56] Takahashi, A. and Yoshida, N. (2004). "An Asymptotic Expansion Scheme for Optimal Investment Problems," Statistical Inference for Stochastic Processes, 7, No.2, 153-188. 
[57] Takahashi, A. and Yoshida, N. [2005], "Monte Carlo Simulation with Asymptotic Method," The Journal of Japan Statistical Society, Vol. 35-2, 171-203.

[58] Toda, M. [2009], "Notes on an Asymptotic Expansion- Applications and Extensions -," Master Thesis, Graduate School Economics, The University of Tokyo, 2009.

[59] Watanabe, S., Analysis of Wiener Functionals (Malliavin Calculus) and Its Applications to Heat Kernels, The Annals of Probability, Vol.15, 1987, 1-39.

[60] Yamamoto, K. Sato, S. and Takahashi, A. [2008], "Probability Distribution and Option Pricing for Drawdown in a Stochastic Volatility Environment," International Journal of Theoretical and Applied Finance, vol.13-2, 335-354, 2010

[61] Yamamoto, K. and Takahashi, A. [2008], "A Remark on a Singular Perturbation Method for Option Pricing under a Stochastic Volatility Model," Asia-Pacific Financial Markets Vol.16-4, 333-345, 2009.

[62] Yoshida, N., Asymptotic Expansion for Small Diffusions via the Theory of Malliavin-Watanabe, Probability Theory and Related Fields, Vol. 92, 1992a, 275-311.

[63] Yoshida, N., Asymptotic Expansions for Statistics Related to Small Diffusions, Journal of Japan Statistical Society, Vol.22, 1992b, 139-159. 
Table 2: Option prices under Black-Scholes model

\begin{tabular}{c|ccc|cc}
\hline strike & $(\mathrm{a})$ & $(\mathrm{b})$ & $(\mathrm{c})$ & $(\mathrm{b})-(\mathrm{a})$ & $(\mathrm{c})-(\mathrm{a})$ \\
\hline 10 & -0.00 & 0.00 & 0.00 & 0.001 & 0.000 \\
20 & 0.00 & 0.01 & 0.00 & 0.005 & -0.000 \\
30 & 0.00 & 0.01 & 0.00 & 0.013 & 0.000 \\
40 & 0.00 & 0.02 & 0.00 & 0.019 & -0.000 \\
50 & 0.00 & 0.01 & 0.00 & 0.011 & -0.002 \\
\hline 60 & 0.04 & 0.04 & 0.04 & -0.006 & -0.006 \\
70 & 0.34 & 0.33 & 0.33 & -0.010 & -0.007 \\
80 & 1.43 & 1.42 & 1.43 & -0.004 & -0.000 \\
90 & 3.99 & 3.99 & 3.99 & -0.001 & 0.002 \\
100 & 8.45 & 8.45 & 8.45 & -0.000 & -0.000 \\
\hline 110 & 4.75 & 4.75 & 4.74 & 0.001 & -0.002 \\
120 & 2.50 & 2.51 & 2.50 & 0.003 & 0.000 \\
130 & 1.25 & 1.26 & 1.26 & 0.009 & 0.006 \\
140 & 0.60 & 0.61 & 0.61 & 0.009 & 0.010 \\
150 & 0.28 & 0.28 & 0.28 & -0.001 & 0.007 \\
\hline 160 & 0.12 & 0.11 & 0.13 & -0.013 & 0.004 \\
170 & 0.05 & 0.04 & 0.06 & -0.017 & 0.002 \\
180 & 0.02 & 0.01 & 0.02 & -0.013 & 0.001 \\
190 & 0.01 & 0.00 & 0.01 & -0.008 & 0.000 \\
200 & 0.00 & 0.00 & 0.00 & -0.004 & 0.000 \\
\hline
\end{tabular}


Figure 1: Densities under Black-Scholes model (total)

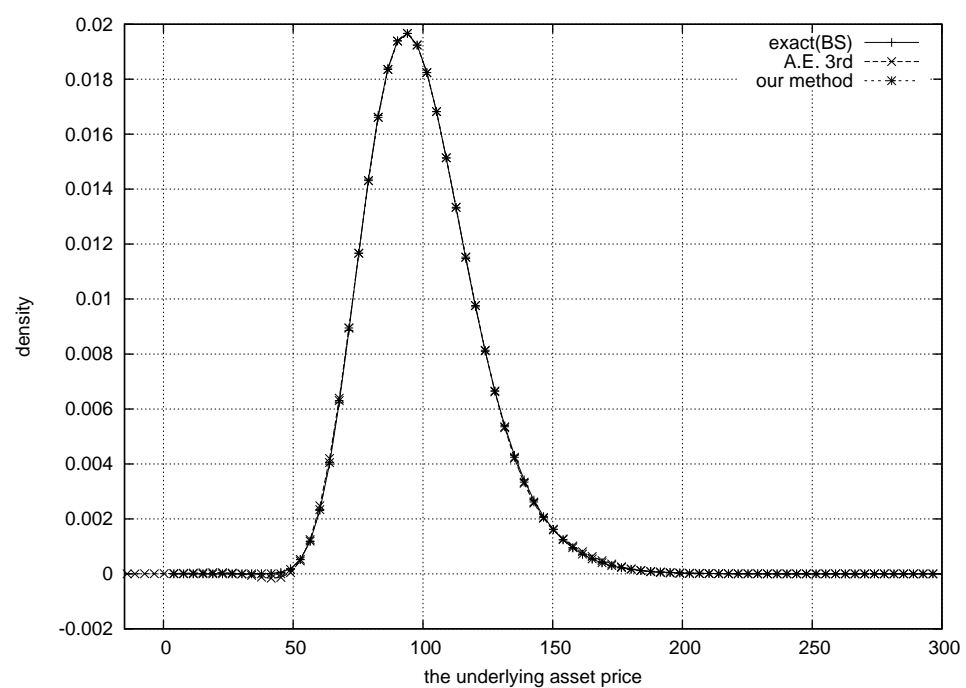

Figure 2: Densities under Black Scholes model (left tail)

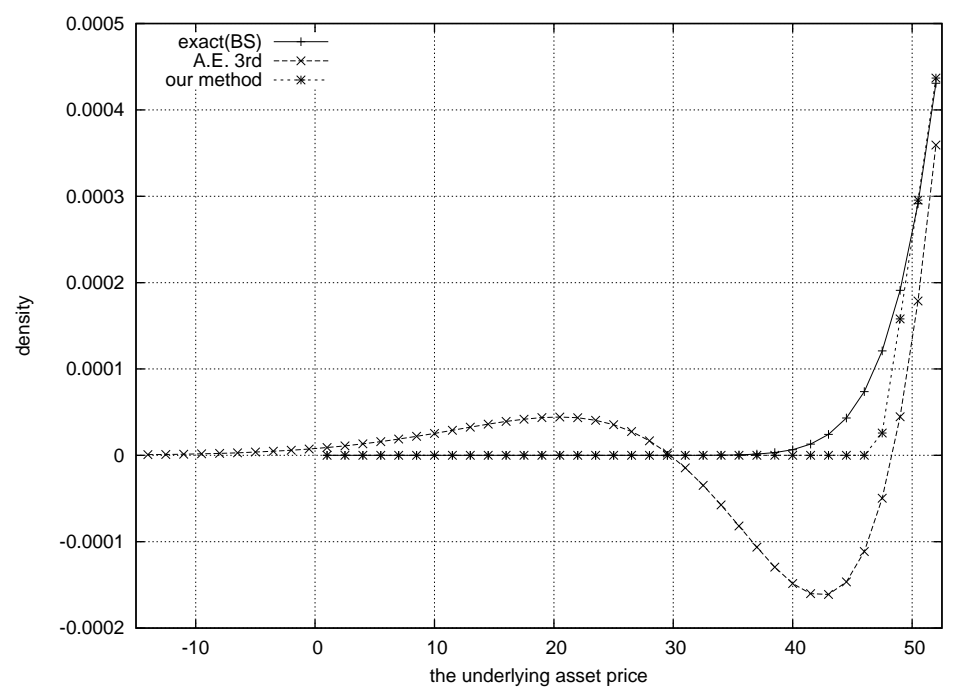


Figure 3: Densities under Black-Scholes model (right tail)

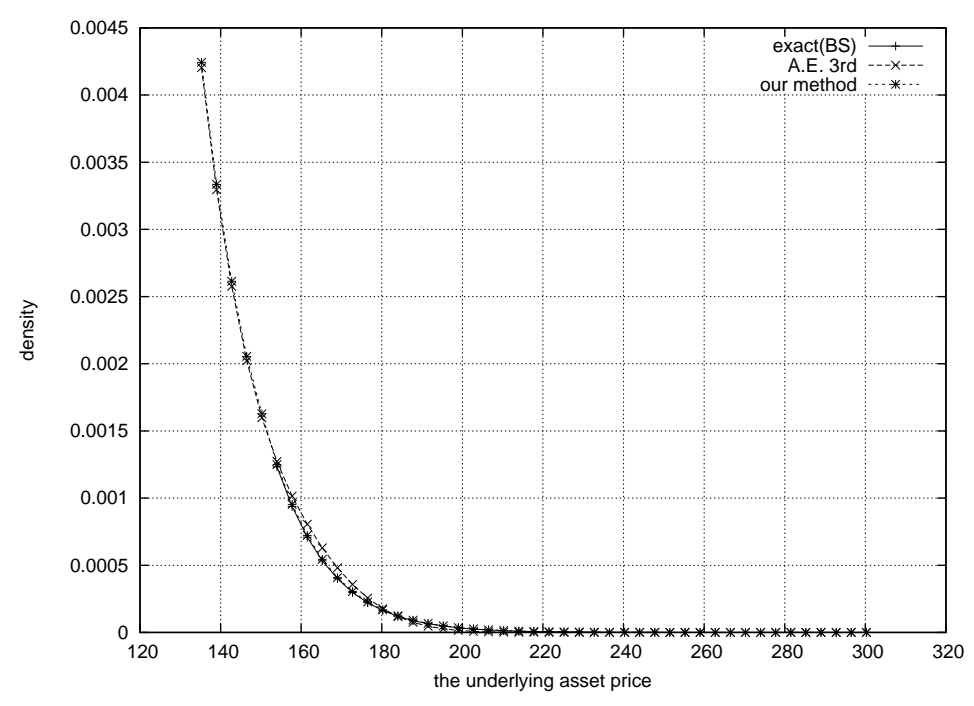


Table 3: Option prices under SABR model with $c=1$

\begin{tabular}{c|cccc|ccc}
\hline strike & $(\mathrm{a})$ & $(\mathrm{b})$ & $(\mathrm{c})$ & $(\mathrm{d})$ & $(\mathrm{b})-(\mathrm{a})$ & $(\mathrm{c})-(\mathrm{a})$ & $(\mathrm{d})-(\mathrm{a})$ \\
\hline 10 & 0.00 & 0.00 & -0.14 & 0.01 & 0.00 & 0.14 & -0.00 \\
20 & 0.05 & 0.03 & -0.13 & 0.06 & 0.02 & 0.18 & -0.02 \\
30 & 0.25 & 0.23 & 0.09 & 0.28 & 0.02 & 0.16 & -0.03 \\
40 & 0.79 & 0.78 & 0.68 & 0.83 & 0.02 & 0.12 & -0.04 \\
50 & 1.86 & 1.86 & 1.80 & 1.93 & 0.00 & 0.06 & -0.06 \\
\hline 60 & 3.64 & 3.64 & 3.61 & 3.71 & -0.00 & 0.03 & -0.07 \\
70 & 6.25 & 6.25 & 6.24 & 6.31 & -0.01 & 0.01 & -0.07 \\
80 & 9.75 & 9.76 & 9.76 & 9.80 & -0.01 & -0.00 & -0.05 \\
90 & 14.17 & 14.17 & 14.17 & 14.20 & -0.01 & -0.01 & -0.03 \\
100 & 19.44 & 19.45 & 19.46 & 19.46 & -0.01 & -0.01 & -0.01 \\
\hline 110 & 15.54 & 15.53 & 15.54 & 15.55 & 0.01 & 0.00 & -0.01 \\
120 & 12.33 & 12.32 & 12.33 & 12.33 & 0.01 & -0.01 & -0.01 \\
130 & 9.72 & 9.72 & 9.74 & 9.73 & 0.01 & -0.02 & -0.01 \\
140 & 7.64 & 7.63 & 7.67 & 7.65 & 0.01 & -0.03 & -0.01 \\
150 & 5.98 & 5.98 & 6.03 & 6.00 & 0.00 & -0.05 & -0.02 \\
\hline 160 & 4.68 & 4.68 & 4.76 & 4.70 & -0.00 & -0.08 & -0.03 \\
170 & 3.65 & 3.65 & 3.76 & 3.68 & -0.00 & -0.11 & -0.03 \\
180 & 2.85 & 2.85 & 2.97 & 2.87 & -0.00 & -0.12 & -0.02 \\
190 & 2.23 & 2.22 & 2.35 & 2.24 & 0.00 & -0.12 & -0.01 \\
200 & 1.74 & 1.73 & 1.84 & 1.72 & 0.01 & -0.10 & 0.02 \\
\hline
\end{tabular}


Figure 4: Densities under SABR model with $c=1$ (total)

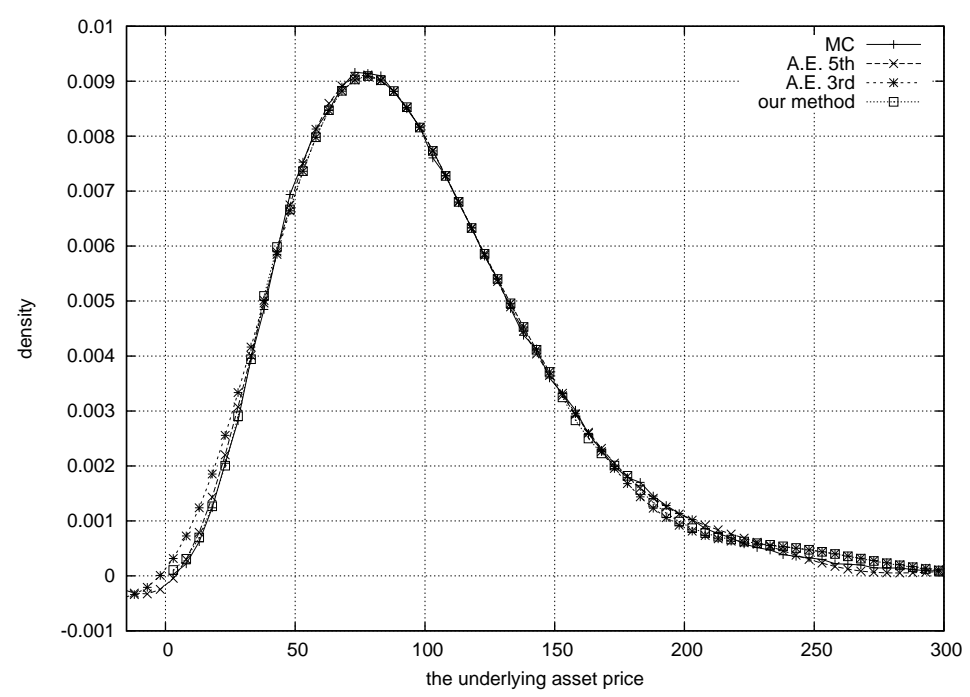

Figure 5: Densities under SABR model with $c=1$ (left tail)

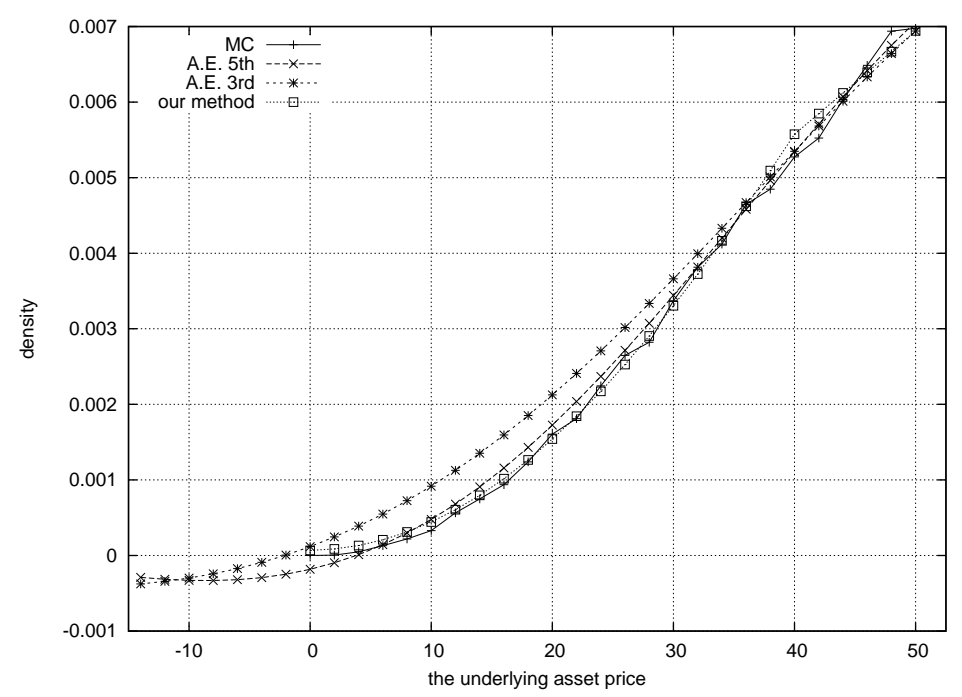


Figure 6: Densities under SABR model with $c=1$ (right tail)

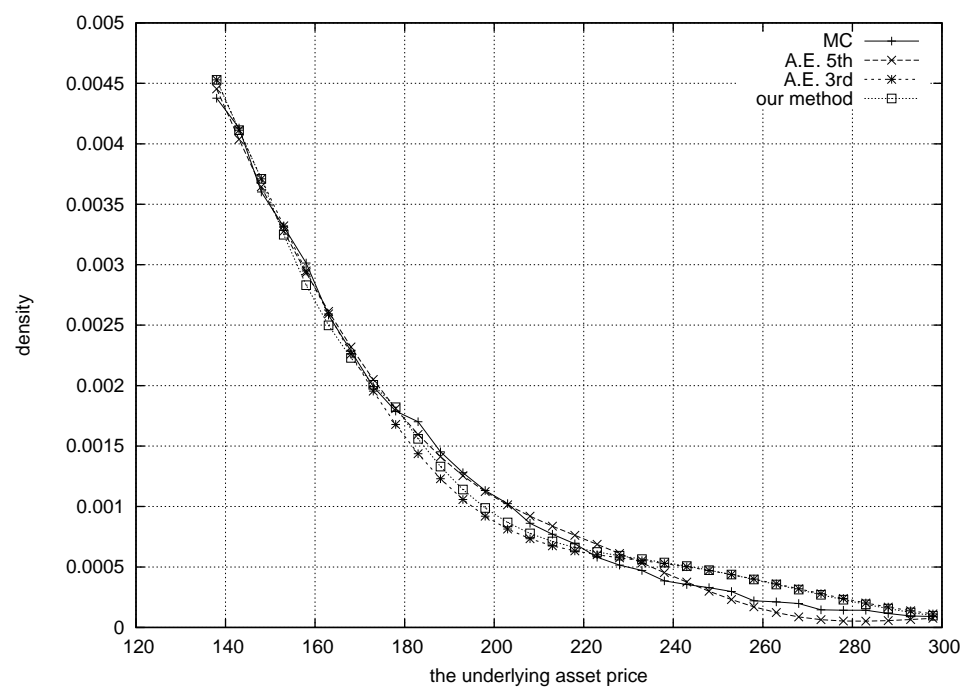


Table 4: Option prices under SABR model with $c=\frac{1}{2}$

\begin{tabular}{c|cccc|ccc}
\hline strike & $(\mathrm{a})$ & $(\mathrm{b})$ & $(\mathrm{c})$ & $(\mathrm{d})$ & $(\mathrm{b})-(\mathrm{a})$ & $(\mathrm{c})-(\mathrm{a})$ & $(\mathrm{d})-(\mathrm{a})$ \\
\hline 10 & 0.13 & 0.15 & 0.28 & 0.15 & -0.02 & -0.15 & -0.02 \\
20 & 0.43 & 0.46 & 0.61 & 0.46 & -0.02 & -0.17 & -0.03 \\
30 & 0.98 & 1.00 & 1.16 & 1.00 & -0.03 & -0.18 & -0.03 \\
40 & 1.86 & 1.89 & 2.04 & 1.88 & -0.03 & -0.18 & -0.02 \\
50 & 3.19 & 3.21 & 3.35 & 3.21 & -0.03 & -0.16 & -0.02 \\
\hline 60 & 5.06 & 5.09 & 5.20 & 5.08 & -0.02 & -0.14 & -0.01 \\
70 & 7.58 & 7.60 & 7.69 & 7.60 & -0.02 & -0.11 & -0.01 \\
80 & 10.82 & 10.84 & 10.91 & 10.84 & -0.02 & -0.08 & -0.02 \\
90 & 14.84 & 14.86 & 14.90 & 14.87 & -0.01 & -0.06 & -0.03 \\
100 & 19.66 & 19.68 & 19.70 & 19.70 & -0.01 & -0.03 & -0.03 \\
\hline 110 & 15.30 & 15.29 & 15.29 & 15.27 & 0.02 & 0.01 & 0.03 \\
120 & 11.67 & 11.66 & 11.64 & 11.64 & 0.01 & 0.03 & 0.03 \\
130 & 8.73 & 8.72 & 8.69 & 8.71 & 0.01 & 0.04 & 0.02 \\
140 & 6.42 & 6.41 & 6.36 & 6.40 & 0.01 & 0.06 & 0.02 \\
150 & 4.64 & 4.62 & 4.56 & 4.62 & 0.01 & 0.08 & 0.01 \\
\hline 160 & 3.29 & 3.28 & 3.21 & 3.28 & 0.01 & 0.09 & 0.01 \\
170 & 2.30 & 2.29 & 2.21 & 2.27 & 0.01 & 0.09 & 0.03 \\
180 & 1.59 & 1.57 & 1.49 & 1.54 & 0.01 & 0.09 & 0.04 \\
190 & 1.08 & 1.07 & 0.99 & 1.03 & 0.01 & 0.09 & 0.05 \\
200 & 0.72 & 0.72 & 0.65 & 0.67 & 0.01 & 0.08 & 0.05 \\
\hline
\end{tabular}


Figure 7: Densities under SABR model with $c=\frac{1}{2}$ (total)

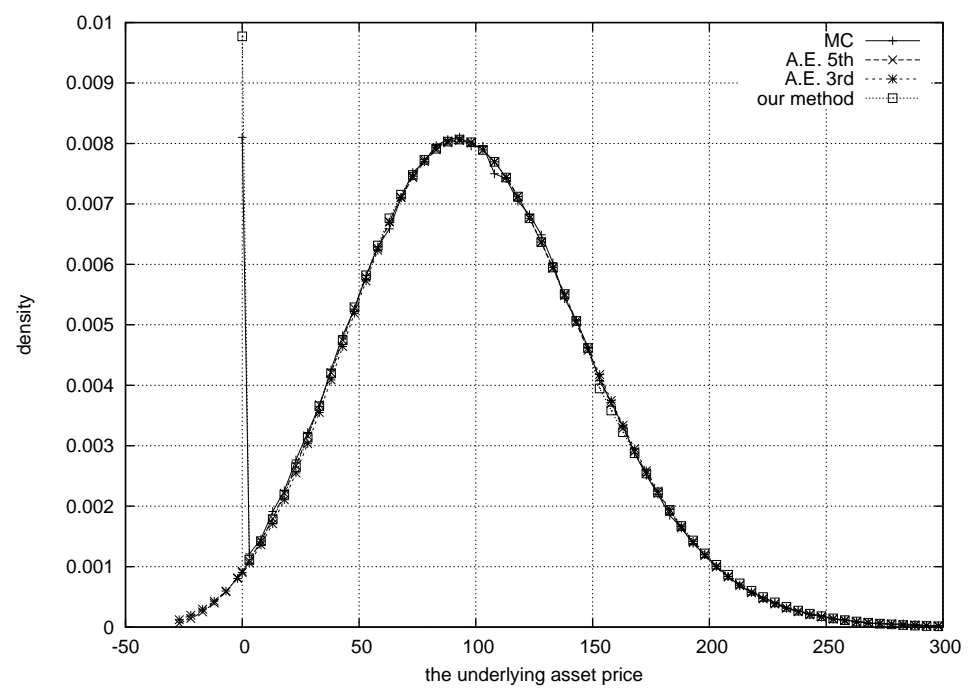

Figure 8: Densities under SABR model with $c=\frac{1}{2}$ (left tail)

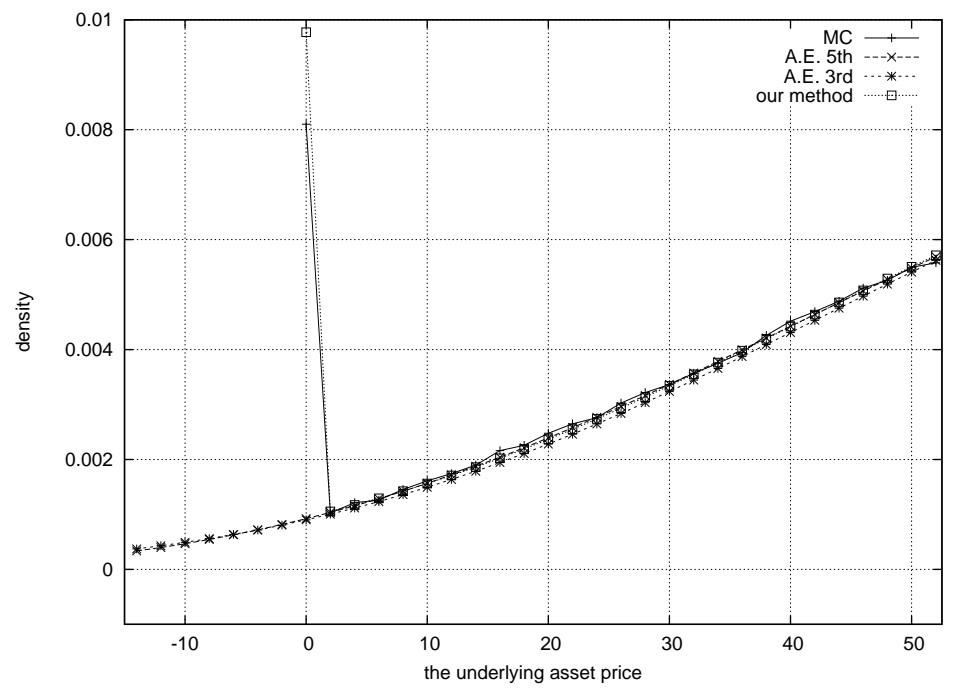


Figure 9: Densities under SABR model with $c=\frac{1}{2}$ (right tail)

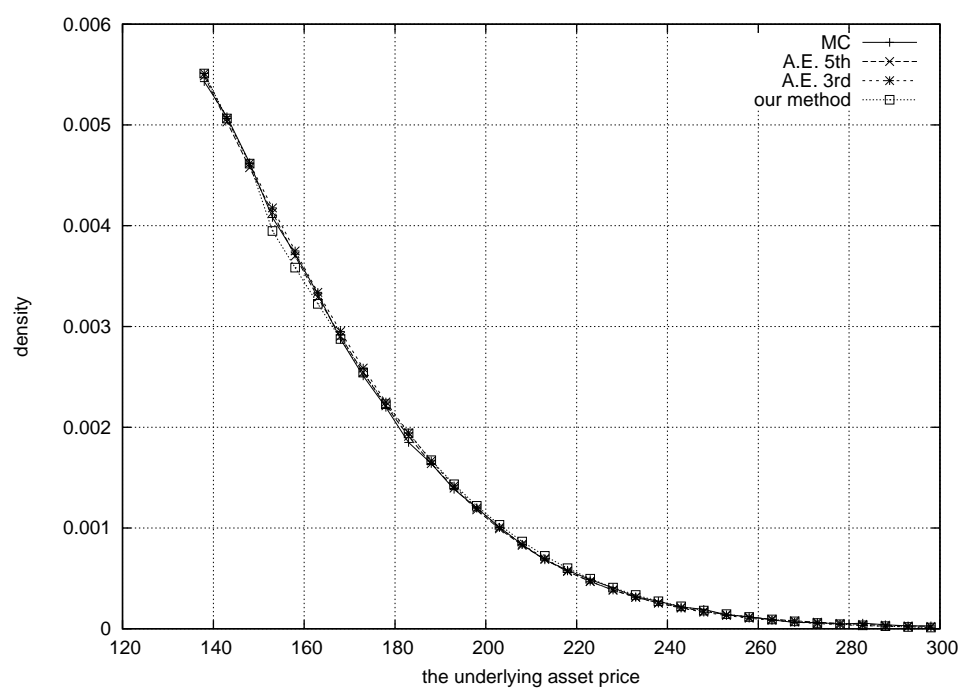

